\title{
Cetoacidosis diabética euglucémica, un diagnóstico difícil de identificar
}

\author{
Euglicemic diabetic ketoacidosis, an easily missed diagnosis
}

\author{
Mário Monteiro-Santos, Telma Elias, Cármen Ferreira, Daniela Brito \\ IInternal Medicine Department. Centro Hospitalar do Médio Tejo. Abrantes. Portugal
}

\begin{abstract}
Diabetic ketoacidosis is a serious and potentially life-threatening acute complication of diabetes mellitus. SGLT-2 inhibitors are recommended as first-line therapy in patients unable to tolerate metformin or as second-line agents after metformin. Their use is increasing as new data show, besides improving glycemic control, weight loss, blood pressure reduction, and beneficial cardiovascular and reno-protective effects. Euglycemic diabetic ketoacidosis is a rare but potential complication of SGLT-2 inhibitors. Physicians including internists, intensivists and emergency physicians should all be aware as this diagnosis can easily be missed in the absence of evident hyperglycemia. We report a case of 61 -year-old male admitted in the emergency room because of altered mental status, associated with holocranial headache. He had medical history of type 2 diabetes and had recently started a SLGT-2 inhibitor. Arterial blood gases showed a severe high anion gap uncompensated metabolic acidosis. Blood and urine ketones were high with normal serum glucose levels. The diagnosis of euglycemic ketoacidosis due to SLGT-2 inhibitor was made.
\end{abstract}

Palabras clave: cetoacidosis euglucémica, iSGLT2.

Keywords: ketoacidosis, euglycemic, iSGLT2.

\section{INTRODUCTION}

Diabetic ketoacidosis (DKA) is a serious and potentially lifethreatening acute complication of diabetes mellitus (DM)'. DKA diagnosis is based on laboratory testing showing hyperglycemia (glucose $>250 \mathrm{mmol} / \mathrm{l}$ ), metabolic acidosis (arterial pH $<$ 7.3 , and serum bicarbonate $<18 \mathrm{mEq} / \mathrm{II}$, a high anion gap as well as the presence of ketone bodies in the blood or urine ${ }^{2}$. Euglycemic diabetic ketoacidosis (EDKA), unlike classic DKA, presents a glucose level $<250 \mathrm{mmol} / L$ and it is recently associated to sodium glucose cotransporter 2 (SGLT-2) inhibitors ${ }^{3}$, a relatively new class of oral anti-diabetic agents which use is rapidly escalating worldwide. It is important for all clinicians to be aware of the risk of developing DKA even in the absence of evident hyperglycemia, as this diagnosis can be easily missed with potentially dangerous outcome.

\section{CASE REPORT}

A61-year-old male with type $2 \mathrm{DM}$ visited the emergency room because of altered mental status, associated with holocranial headache. The patient had no other chronic disease and was medicated with metformin $1000 \mathrm{mg}$ twice a day, gliclazide $60 \mathrm{mg}$ once a day and canaglifozin $100 \mathrm{mg}$, this one started 5 days prior admission. He had no history of alcohol intake or herbal, illicit drugs exposure. At admission, the patient was confused but cooperative with no focal neurological deficits. Vital signs were $36.7^{\circ} \mathrm{C}$ body temperature, blood pressure 153/72 mmHg, pulse rate was 119 beats per minute, was tachypneic with 23/min respiratory rate, with normal heart sounds and pulmonary auscultation. Blood glucose level was $157 \mathrm{mg} / \mathrm{dl}$. Arterial blood gases (ABG) showed a high anion gap uncompensated metabolic acidosis (pH: 7.21, C02: 22 $\mathrm{mmHg}, \mathrm{HCO}: 9.6 \mathrm{mmol} / \mathrm{L}$, serum lactate $1.7 \mathrm{mmol} / \mathrm{L}$, anion gap: $22.4 \mathrm{mEq} / \mathrm{L})$. Blood and urine ketones were high (8.0 $\mathrm{mmol} / \mathrm{L}$ and $150 \mathrm{mg} / \mathrm{dl}$ respectively). Renal function was preserved, with no electrolyte disturbances (Table 1).
Other causes of increased anion gap metabolic acidosis were excluded. The diagnosis of EDKA was made and insulin infusion therapy, intravenous hydration with $5 \%$ dextrose fluid considering blood glucose and bicarbonate were initiated. The patient was admitted to intensive care unit where in 2 days the EDKA had completely resolved with normalization of acidosis, blood ketones and anion gap. He was then transferred to Internal Medicine ward and discharged home after 3 days without the SGLT-2 inhibitor.

\section{DISCUSSION}

Table 1. Admission laboratory tests

\begin{tabular}{|l|c|c|}
\hline Laboratory tests (units) & Patient's values & Reference value \\
\hline Random blood sugar (mg/dl) & 157 & $65-100$ \\
\hline Hemoglobin (g/dl) & 15,2 & $13-17$ \\
\hline White blood cells (10^9/L) & 10,2 & $4,0-10,0$ \\
\hline Platelets (10^9/L) & 211 & $150-400$ \\
\hline Sodium (mmol/L) & 139 & $136-144$ \\
\hline Potassium (mmol/L) & 3,9 & $3,5-5,1$ \\
\hline Chloride (mmo//L) & 107 & $98-107$ \\
\hline Anion Gap (mEq/L) & 22,4 & \\
\hline Blood Ketones (mmol/L) & 8 & \\
\hline Urea (mg/dl) & 87 & $74-106$ \\
\hline Creatinine (mg/dl) & 1,2 & $0,7-1,2$ \\
\hline Arterial Blood Gas: & & \\
\hline pH & 7,21 & $7,35-7,45$ \\
\hline Bicarbonate & 9,6 & $21,0-28,0$ \\
\hline p02 (mmHg) & 86 & $83-108$ \\
\hline pC02 (mmHg) & 22 & $35-48$ \\
\hline Lactates $(\mathrm{mmol} / \mathrm{L})$ & 1,7 & $0,0-1,3$ \\
\hline Urine Ketones $(\mathrm{mg} / \mathrm{dl})$ & 150 & $<5,0$ \\
\hline Urine Glucose $(\mathrm{mg} / \mathrm{dl})$ & 1000 & $<30,0$ \\
\hline
\end{tabular}


SGLT-2 inhibitors are recommended as first-line therapy in patients unable to tolerate metformin or as second-line agents after metformin ${ }^{1,4}$. They act by inhibiting the SGLT-2 that are expressed in the brush border membrane of the renal proximal tubular cells and accounts for $90 \%$ of total renal glucose absorption, increasing urinary glucose excretion ${ }^{5}$.

Their use is increasing as new data show, besides improving glycemic control, weight loss, blood pressure reduction, and beneficial cardiovascular and reno-protective effects. It is expected that SLGT-2 inhibitors use will only increase over time ${ }^{6}$. They are generally well tolerated but have several well-recognized adverse effects that should be considered to optimize their risk to benefit ratio?.

Ketoacidosis is an adverse effect of SGLT-2 inhibitors use in patients with both type 1 and more rarely type 2 diabetes. Classically is defined as a presence of the triad of hyperglycemia (glucose $>250 \mathrm{mmol} / \mathrm{l}$ ), metabolic acidosis (arterial pH < 7.3, and serum bicarbonate $<18 \mathrm{mEq} / \mathrm{l})$, a high anion gap as well as the presence of Ketone bodies in the blood or urine? Our patient had ketoacidosis, yet his blood glucose level was far below the usual for the "traditional" DKA. The absence of elevated serum glucose levels can delay the recognition and diagnosis of this life-threatening state by the clinician that can lead to a potentially fatal outcome or longer hospital/intensive care unit (ICU) stay ${ }^{8}$.

The mechanism of EDKA ${ }^{9}$ has not been fully elucidated. Some authors propose that by increasing urinary glucose excretion, which in turn reduces insulin secretion from pancreatic $\beta$-cells, results in a lowering of the anti-lipolytic activity of insulin and consequent stimulation of the production of free fatty acids. These free fatty acids are then converted to ketone bodies by $\beta$-oxidation in the liver. Also, there is recent evidence demonstrating the presence of SGLT-2 inhibitors in pancreatic a-cells which directly stimulates glucagon secretion, with further reduction of the insulin-to-glucagon ration, contributing to ketogenesis.

To minimize DKA episodes associated with SGLT-2 inhibitors, prescribing these agents should be avoided in DKA-prone patients, including those with type $1 \mathrm{DM}$, diabetes-related autoimmunity and those with previous history of DKA. Stopping SGLT2 inhibitors should be considered if patients are under conditions that can precipitate DKA ${ }^{3,7,9}$.

Once recognized, the treatment of EDKA is similar to that of DKA and involves rapid correction with intravenous fluids, correction of electrolyte abnormalities and insulin drip with a dextrose solution until anion gap and acidosis normalize ${ }^{10}$. The authors pretend to alert to the possibility of having ketoacidosis with normal glucose levels, which is rare, easy to miss and difficult to diagnosis if not considered, mainly when there is history of SGLT-2 inhibitor use. These patients despite the absence of hyperglycemia should be analyzed with blood gas samples and blood and urine ketones checked.

\section{REFERENCES}

1. Karakaya Z, Topal FE, Topal F, Payza U, Akyol PY. Euglisemic diabetic ketoacidotic coma caused by dapagliflozin. Am J Emerg Med. 2018;36(11):2136.e1-2136.e2.

2. Chiasson JL, Aris-Jilwan N, Bélanger R, et al. Diagnosis and treatment of diabetic ketoacidosis and the hyperglycemic hyperosmolar state [published correction appears in CMAJ. 2003 May 13;168(10):1241]. CMAJ. 2003;168(7):859-866.

3. Goldenberg RM, Berard LD, Cheng AYY, et al. SGLT2 Inhibitor-associated Diabetic Ketoacidosis: Clinical Review and Recommendations for Prevention and Diagnosis. Clin Ther. 2016;38(12):2654-2664.e1.

4. American Diabetes Association: standards on medical care in diabetes - 2016 Diabetes Care. 2016;39(suppl 1):S1-110

5. van Bommel EJ, Muskiet MH, Tonneijck L, Kramer MH, Nieuwdorp M, van Raalte DH. SGLT2 Inhibition in the Diabetic Kidney-From Mechanisms to Clinical Outcome. Clin J Am Soc Nephrol. 2017;12(4):700-710.

6. Rabizadeh S, Nakhjavani M, Esteghamati A. Cardiovascular and Renal Benefits of SGLT2 Inhibitors: A Narrative Review. Int J Endocrinol Metab. 2019;17(2):e84353.

7. Lupsa BC, Inzucchi SE. Use of SGLT2 inhibitors in type 2 diabetes: weighing the risks and benefits. Diabetologia. 2018;61(10):2118-2125.

8. Burke KR, Schumacher CA, Harpe SE. SGLT2 Inhibitors: A Systematic Review of Diabetic Ketoacidosis and Related Risk Factors in the Primary Literature. Pharmacotherapy. 2017;37(2):187-194.

9. Barski L, Eshkoli T, Brandstaetter E, Jotkowitz A. Euglycemic diabetic ketoacidosis. Eur J Intern Med. 2019;63:9-14.

10. Dizon S, Keely EJ, Malcolm J, Arnaout A. Insights Into the Recognition and Management of SGLT2-Inhibitor-Associated Ketoacidosis: It's Not Just Euglycemic Diabetic Ketoacidosis. Can J Diabetes. 2017;41(5):499-503. 